\title{
Características químicas y sensoriales de la carne de cerdo, en función del consumo de dietas con ractopamina y diferentes concentraciones de lisina
}

\section{Effect of ractopamine and different lysine concentrations in pig diets on pork chemical and sensory characteristics}

\author{
María Antonia Mariezcurrena-Berasaina, Diego Braña-Varelab, María Dolores \\ Mariezcurrena-Berasainc, Ignacio Arturo Domínguez-Vara a, Danilo Méndez-Medinad, \\ María Salud Rubio-Lozanod
}

\begin{abstract}
RESUMEN
Se evaluaron las características químicas y sensoriales de la carne de cerdos alimentados con Ractopamina (RAC) a 5 ppm (Paylean ${ }^{\circledR}$, Elanco, México) y diferentes concentraciones de lisina digestible ileal estandarizada (lys-dig), en dietas con 3.3 Mcal EM/ kg y $14.5 \%$ de proteína cruda, lo que representa un contenido de proteína de $10 \%$ inferior al mínimo recomendado por el laboratorio y la literatura. Se utilizaron 48 cerdos (peso corporal inicial $77.2 \pm 3.42$ y final $110.0 \pm 3.0 \mathrm{~kg}$ ) que fueron asignados a uno de cuatro tratamientos: C) Dieta de finalización Control (lys-dig 0.65\%); RAL) alta en Lisina con RAC (lys-dig 1\%); RNL) Niveles normales de lisina con RAC (lis-dig $0.80 \%$ ); RBL) Baja en lisina con RAC (lis-dig $0.50 \%$ ). Después de 28 días en sus respectivas dietas, los animales se sacrificaron y procesaron. De cada animal, se recolectaron cuatro chuletas $(2.5$ cm de grosor) y se determinó grasa intramuscular y evaluación sensorial. Los resultados indican que con la adición de RAC, en dietas con $14.5 \%$ de proteína, se aumenta $(P<0.01)$ la grasa intramuscular en $25 \%$. Un incremento mayor (hasta el $50 \%)$ en grasa intramuscular se obtuvo con dietas con RAC y una baja concentración de lisina ( $0.5 \%$ lys-dig). El panel de consumidores no detectó diferencias en aroma, sabor y textura $(P>0.17)$, pero sí detectó una mayor jugosidad de carne de cerdo $(P<0.02)$ de animales que consumieron la dieta $R B L$. Es factible mejorar la calidad de la carne de cerdo mediante el uso de RAC y dietas bajas en proteína y lisina.
\end{abstract}

PAlabras ClaVE: Carne de cerdo, Beta adrenérgico, Composición química, Análisis sensorial.

\begin{abstract}
Pork chemical and sensory characteristics of 48 barrows were used to evaluate the effect of ractopamine (5 PPM, Paylean, Elanco México) in diets formulated to three standardized ileal digestible-lysine (SID-Lys) levels. All diets had $3.3 \mathrm{Mcal}$ EM/kg, and 14.5 \% crude protein (CP), this represent a $10 \%$ lower $\mathrm{CP}$ content than that recommended by the laboratory and the literature. Pigs (initial BW 77.2 \pm 3.42 and final $B W 110.0 \pm 3.0 \mathrm{~kg}$ ) were randomly alloted to one of four treatments: C) Control diet, $0.65 \%$ SID-Lys; RHL) ractopamine, high lysine level, $1 \%$ SID-Lys; RNL) ractopamine, normal lysine level, $0.8 \%$ SID-Lys; RLL) ractopamine, low lysine level, $05 \%$ SID-Lys. After $28 \mathrm{~d}$ of ad libitum intake, pigs were killed; from each pig, four $2.5 \mathrm{~cm}$ thick chops were used for chemical and sensory analyses. Adding RAC in diets with $14.5 \% \mathrm{CP}$ increases $(P<0.01)$ intramuscular fat in $25 \%$. A higher increase (until $50 \%$ ) was achieved when RAC was used in a diet with a low SID-Lys. Pork chop consumer acceptance was evaluated using a panel that consisted of 60 consumers. They were not able to detect differences in texture, flavor and aroma $(P>0.17)$, but they detected a higher juiciness $(P<0.02)$ in chops from pigs fed the RLL diet. By improving marbling and juiciness, RAC-containing diets supplemented with low levels of crude protein and low SID-Lys can improve pork quality.
\end{abstract}

KEY WORDS: Pork, B-adrenergic, Chemical composition, Sensory analysis.

\footnotetext{
Recibido el 7 de junio de 2011. Aceptado el 11 de noviembre de 2011.

a Departamento de Nutrición Animal, Facultad de Medicina Veterinaria y Zootecnia, Universidad Autónoma del Estado de México.

b CENID-Fisiología, Instituto Nacional de Investigaciones Forestales Agrícolas y Pecuarias. Km 1 Carretera a Colón, C.P. 76280. Ajuchitlán, Qro., México. brana.diego@inifap.gob.mx. Correspondencia al segundo autor.

c Departamento de Tecnología de los Alimentos, Facultad de Ciencias Agrícolas, Universidad Autónoma del Estado de México.

d Centro de Enseñanza, Práctica e Investigación en Producción y Salud Animal. Facultad de Medicina Veterinaria y Zootecnia, Universidad Nacional Autónoma de México.
} 


\section{INTRODUCCIÓN}

Una alternativa para aumentar la competitividad, es la obtención de productos diferenciados. En la carne de cerdo, la grasa intramuscular (GIM) puede ser un factor de diferenciación de producto a nivel comercial(1) que influye positivamente en la

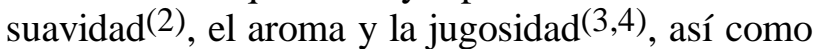
en la aceptación general de la carne ${ }^{(4,5)}$.

Además de la genética( ${ }^{(6)}$, otra forma de incrementar el contenido de GIM en la carne de cerdo es mediante el suministro de dietas deficientes en proteína durante la fase final de la engorda $(7,8)$, especialmente cuando la concentración de lisina es baja $(9,10)$. Sin embargo, estas dietas reducen la eficiencia alimenticia y la tasa de crecimiento(11).

El clorhidrato de ractopamina (RAC, Paylean ${ }^{\circledR}$, Elanco una división de Eli Lilly Co., México) es una fenatolamina con actividad agonista $\beta$-adrenérgica usada como promotor del crecimiento, que mejora la eficiencia alimenticia y el rendimiento de la canal( $^{(12,13)}$. Smith ${ }^{(14)}$ sugiere que la RAC promueve que los nutrientes sean orientados más enfáticamente a la síntesis de proteína muscular, además de que reduce la tasa de degradación de proteínas, lo que acaba promoviendo la hipertrofia muscular(15).

El laboratorio que produce la RAC en forma comercial, así como diversos estudios $(16,17)$, recomiendan su uso con dietas que contengan un mínimo de $16 \%$ de proteína cruda (PC) para alcanzar la concentración adecuada de aminoácidos que sustenten el desarrollo muscular. Sin embargo, otros autores $(18,19)$ sugieren que los efectos de la RAC pueden ser afectados más por la restricción de energía, que por la de proteína.

En un meta-análisis realizado por el grupo de Apple(20), al analizar los efectos de la RAC en cerdos, concluyen que la mayor parte de la información analizada ha fallado en detectar diferencias en la calidad de la carne, particularmente en los valores de marmoleo cuando se comparan dietas con o sin RAC, lo cual pudiera estar asociado a la variación en los sistemas de formulación de

\section{INTRODUCTION}

Differentiated meat products are an alternative for increasing competitiveness. On a commercial level,(1) intramuscular fat (IMF) can be a differentiating factor in pork because it positively affects tenderness ${ }^{(2)}$, aroma and juiciness $(3,4)$, as well as general acceptance $(4,5)$. Besides genetics $(6)$, IMF can also be elevated by administering protein deficient diets in the final stage of the fattening period $(7,8)$, particularly at low lysine concentrations $(9,10)$. However, these diets reduce feed efficiency and growth rate(11).

Ractopamine hydrochloride (RAC, Paylean ${ }^{\circledR}$, Elanco, a division of Eli Lilly Co., Mexico) is a phenatolamine with $\beta$-adrenergic agonist activity. It is used as a growth promoter which improves feed efficiency and carcass yield(12,13). Smith(14) suggests that RAC promotes a more emphatic use of nutrients towards muscular protein synthesis, as well as reducing the protein degradation rate, which boosts muscular hypertrophy(15). Both, the manufacturer and different authors $(16,17)$, recommend RAC to be applied in diets containing a minimum of $16 \%$ crude protein $(\mathrm{CP})$ to ensure a sufficient amount of amino acids concentration to support muscular development. However, other authors believe that the effect of RAC is more efficient by dietary energy restriction than protein levels $(18,19)$.

In a meta-analysis of the effects of RAC in pigs, Apple et al ${ }^{(20)}$ concluded that most of the analyzed data indicate no differences in meat quality, particularly in marbling, when comparing diets with and without RAC. They suggest this may be due to variation in formulation systems between studies. For example, Webster(17) states that both lysineassociated protein concentration and $\mathrm{RAC}$, be it separately or in combination, can change carcass characteristics by reducing subcutaneous fat deposition.

The present study objective was to evaluate if chemical and sensory changes, mainly marbling, in 
cada experimento. Por ejemplo, Webster(17) indica que por separado o en combinación, la concentración de proteína (asociada a lisina) y de RAC alteran las características de la canal al reducir el depósito de grasa subcutánea.

En el presente estudio, se propuso la hipótesis de que al suministrar dietas con RAC, los cambios en las características químicas y sensoriales de la carne de cerdo, principalmente en el marmoleo, serán una consecuencia de la concentración de la lisina dietaria usada. Contrario a las concentraciones altas, las dietas bajas en lisina deben promover un mayor marmoleo en la carne de cerdos en finalización.

\section{MATERIALES Y MÉTODOS}

Animales, manejo y alimentación

Para evaluar los efectos de la RAC y de diferentes concentraciones de lisina sobre las características pork from pigs fed diets containing RAC is a consequence of dietary lysine concentration. Low lysine concentrations can be expected to promote more marbling in finishing pigs.

\section{MATERIALS AND METHODS}

Animals, handling and feeding

To evaluate the effects of RAC and different dietary lysine concentration over the chemical and sensory characteristics of pork, an experiment was set with 48 barrows (experimental unit) randomly housed in individual pens $\left(1.25 \mathrm{~m}^{2}\right)$. Animals were the progeny of 337PIC line sires (PIC, Franklin, KY, USA) crossed with Landrace $\times$ Duroc sows. Experimental diets were administered during the last $28 \mathrm{~d}$ of the growth period: initial live weight= $77.2 \pm 3.4 \mathrm{~kg}$, age $=128 \pm 3 \mathrm{~d}$; final live weight $=$ $110 \pm 3.0 \mathrm{~kg} ;$ age $=156 \pm 3 \mathrm{~d}$

Cuadro 1. Composición de las dietas ( $\mathrm{kg} \mathrm{t}^{-1}$ en base húmeda)

Table 1. Diet composition ( $\mathrm{kg} \mathrm{t}^{-1}$ wet base) supplied to pigs during 28 days

\begin{tabular}{lcccr}
\hline & \multicolumn{4}{c}{ Experimental Diets } \\
\cline { 2 - 5 } Ingredient & $\mathrm{C}^{*}$ & $\mathrm{RHL}$ & $\mathrm{RNL}$ & $\mathrm{RLL}$ \\
\hline Sorghum & 813.64 & 610.25 & 750.59 & 788.81 \\
Soy paste $(46 \% \mathrm{CP})$ & 133.03 & 339.17 & 195.73 & 20.88 \\
Corn gluten & 0 & 0 & 0 & 100.00 \\
Soy hulls & 0 & 0 & 0 & 53.34 \\
Vegetable oil & 30.00 & 30.00 & 30.00 & 15.00 \\
Calcium carbonate & 7.57 & 5.99 & 7.09 & 8.16 \\
Dicalcium phosphate & 5.36 & 6.06 & 5.58 & 4.05 \\
L-Lysine. HCl & 2.17 & 0 & 2.07 & 2.81 \\
Salt (NaCl) & 3.50 & 3.50 & 3.50 & 3.50 \\
Vitamins & 2.40 & 2.40 & 2.40 & 2.40 \\
L-Threonine & 1.27 & 1.09 & 1.53 & 0 \\
DL-Methionine & 0.26 & 0.49 & 0.46 & 0 \\
Trace minerals§ & 0.80 & 0.80 & 0.80 & 0.80 \\
Ractopamine-HCl & 0 & 0.25 & 0.25 & 0.25 \\
\hline
\end{tabular}

$\mathrm{C}=\mathrm{Control}$, normal lysine level $(0.65 \%$ standardized ileal digestible lysine); $\mathrm{RHL}=$ ractopamine $(5 \mathrm{ppm})$, high lysine level $(1 \%) ; \mathrm{RNL}=$ ractopamine, normal lysine level $(0.8 \%) ; \mathrm{RLL}=$ ractopamine, low lysine level $(0.5 \%)$.

† Vitamin premix; each $\mathrm{kg}$ diet provided: 2,200 mg retinol acetate; $16.5 \mathrm{mg}$ colecalciferol; $80 \mathrm{mg}$ DL-tocoferol acetate; $4.4 \mathrm{mg}$ menadione sodium bisulphite; $242 \mathrm{mg}$ choline; $33 \mathrm{mg}$ niacin; $24.2 \mathrm{mg}$ D-pantothenic acid; $8.8 \mathrm{mg}$ riboflavin; and $0.04 \mathrm{mg}$ vitamin B12.

$\S$ Mineral premix; each kg diet provided: 70 mg Fe; 70 mg Zn; 27 mg Mn; 8 mg Cu; 0.38 mg I; 0.2 mg Se. 
químicas y sensoriales de la carne de cerdo, se utilizaron 48 cerdos machos castrados, alojados en corrales individuales de $1.25 \mathrm{~m}^{2}$ previa aleatorización. La progenie fue originada con la línea de sementales 337 PIC (PIC, Franklin, KY) cruzados con hembras Landrace $\times$ Duroc. Las dietas experimentales se proporcionaron durante los últimos 28 días del período de engorda; el peso vivo inicial fue de $77.2 \pm 3.4 \mathrm{~kg}$ y la edad de 128 \pm 3 días, el peso vivo final fue de $110 \pm 3.0 \mathrm{~kg}$ y la edad de $156 \pm 3$ días.

Cuatro dietas experimentales (Tratamientos, Cuadro 1) fueron formuladas para ser isocalóricas (3.3 Mcal $\mathrm{EM} / \mathrm{kg}$ ), con un contenido similar de proteína (14.5 $\%), \mathrm{Ca}(0.45 \%)$ y $\mathrm{P}$ disponible $(0.16 \%)$; excepto por el tratamiento control, todas las demás incluyeron $5 \mathrm{ppm}$ de RAC. Las dietas fueron las siguientes: C) Control: normal en lisina ( $0.65 \%$ de lisina digestible ileal estandarizada); RAL) Ractopamina alta en lisina ( $1 \%$ lisina); RNL) Ractopamina normal en lisina (0.8\% de lisina); RBL) Ractopamina baja en lisina ( $0.5 \%$ de lisina).

Las dietas estuvieron basadas en el uso de sorgo y soya. En la dieta con baja concentración de lisina se utilizó gluten de maíz como fuente de proteína de buena calidad (por su elevada concentración de leucina) pero baja en lisina. El contenido de minerales y vitaminas fue suficiente para cubrir los requerimientos de los cerdos $(21)$ y la concentración media de EM en las dietas fue de $3.3 \mathrm{Mcal} \mathrm{kg}^{-1}$ (Cuadro 2).

Las concentraciones de lisina digestible se establecieron como el requerimiento de la población en estudio y según la respuesta esperada para inducir crecimiento magro en cerdos tratados con 5 ppm de RAC ${ }^{(22)}$. Además de la lisina, se verificó que la concentración de los aminoácidos limitantes fuera adecuada para cumplir con las recomendaciones de un patrón de proteína ideal(23).

La faena de los cerdos y el despiece de las canales se realizaron, respectivamente, en el establecimiento TIF (rastro tipo inspección federal) FYRASA
Experimental diets (Table 1) were formulated to be isocaloric (3.3 Mcal ME/kg) and to provide similar levels of protein (14.5\%), $\mathrm{Ca}(0.45 \%)$ and available P $(0.16 \%)$. All diets, except the Control, included RAC at $5 \mathrm{ppm}$, and were formulated as follows: C, a control with normal lysine content (0.65\% standardized ileal digestible lysine); RHL, 5 ppm RAC, high (1\%) lysine content; RNL, 5 ppm RAC, normal $(0.8 \%)$ lysine content; and RLL, 5 ppm RAC, low (0.5\%) lysine content.

Diets were based on sorghum and soybean meal. Corn gluten was used as a protein source in the RLL due to its high leucine and low lysine content. All diets contained vitamin and mineral content sufficient to cover the requirements of growing pigs (21), and mean metabolizable energy (ME) of 3.3 Mcal kg-1 (Table 2). Digestible lysine concentrations were established based on study population requirements and the expected response for lean growth in pigs receiving 5 ppm RAC(22). Limiting amino acid concentration was confirmed to meet recommendations for an ideal protein pattern(23).

On d 29 of the experimental period, feed was not provided for $8 \mathrm{~h}$, and then pigs were weighed and transported $(2 \mathrm{~h})$ to the slaughterhouse, kept in lairage for $6 \mathrm{~h}$ and finally slaughtered. Slaughter was carried out at the federally inspected (TIF) slaughterhouse FYRASA (Pénjamo, Guanajuato, Mexico). Carcasses were refrigerated $\left(1^{\circ} \mathrm{C}\right)$ for

Cuadro 2. Composición química de las dietas experimentales (\% base seca)

Table 2. Chemical composition of diets (\% dry base)

\begin{tabular}{lrrrr}
\hline Nutrients & \multicolumn{1}{c}{$\mathrm{C}$} & $\mathrm{RHL}$ & $\mathrm{RNL}$ & $\mathrm{RLL}$ \\
\hline ME, Mcal kg-1 & 3.30 & 3.35 & 3.32 & 3.25 \\
Crude protein, \% & 14.00 & 14.81 & 14.50 & 14.95 \\
Lysine, \% & 0.65 & 1.00 & 0.80 & 0.50 \\
Total Leucine, \% & 1.56 & 2.03 & 1.70 & 2.17 \\
\hline
\end{tabular}

$\mathrm{C}=$ Control, normal lysine level; $\mathrm{RHL}=$ ractopamine, high lysine level; $\mathrm{RNL}=$ ractopamine, normal lysine level; $\mathrm{RLL}=$ ractopamine, low lysine level; $\mathrm{ME}=$ metabolizable energy.

* Standardized ileal digestible lysine. 
ubicado en Pénjamo, y en el Obrador TIF Apaseo, en Apaseo el Grande, Guanajuato.

En el día 29 del periodo experimental, los cerdos se ayunaron $8 \mathrm{~h}$ y fueron pesados y transportados $(2 \mathrm{~h})$ al rastro, donde luego de $6 \mathrm{~h}$ de descanso fueron sacrificados. Las canales permanecieron $24 \mathrm{~h}$ en refrigeración $\left(1^{\circ} \mathrm{C}\right)$, luego de lo cual, de cada cerdo se extrajo el músculo Longissimus dorsi, de donde se cortaron cuatro chuletas $(2.5 \mathrm{~cm}$ de grosor) de entre la octava y doceava costillas, las cuales fueron identificadas, empacadas al vacío y congeladas a $-18{ }^{\circ} \mathrm{C}$ para su posterior análisis químico y sensorial.

\section{Análisis químico}

De cada cerdo, dos chuletas correspondientes a la novena y décima costilla, con $2.5 \mathrm{~cm}$ de grosor, se homogeneizaron mediante un procesador de alimentos (DPA2; Moulinex, Ecually, Francia), y utilizadas para el análisis de humedad (a $85^{\circ} \mathrm{C}$ por 24 h), proteína cruda (método Kjeldahl) y grasa intramuscular (GIM), utilizando una mezcla de cloroformo-metanol 2:1, en un equípo gravimétrico (Soxtec Foss Tecator 2055 gravimeter; adaptado del método oficial AOAC \#991(24,25).

\section{Evaluación sensorial}

Utilizando la metodología propuesta por Pedrero(26), se evaluó el nivel de aceptación de la carne de cerdo, con 60 consumidores (30 hombres y 30 mujeres de entre 20 y 45 años de edad) en la Facultad de Medicina Veterinaria y Zootecnia de la Universidad Autónoma del Estado de México. Antes de iniciar las evaluaciones, a los panelistas se les dieron instrucciones precisas para realizar el análisis y llenar los formatos con escalas hedónicas, luego de lo cual, la prueba inició a las $1100 \mathrm{~h}^{(27)}$. Las variables evaluadas fueron: jugosidad, textura, sabor $\mathrm{y}$ aroma u olor(26).

Un día antes de la evaluación sensorial, la onceava y doceava chuletas de cada cerdo fueron descongeladas durante $20 \mathrm{~h}$ a temperatura de refrigeración y mantenidas en oscuridad hasta que
$24 \mathrm{~h}$. After that, carcasses were cut at the TIF processing center "Obrador TIF Apaseo" (Apaseo el Grande, Guanajuato, Mexico). The Longissimus dorsi was extracted from each carcass and four chops $(2.5 \mathrm{~cm}$ thick) obtained from the eighth to the twelfth ribs. Samples were labeled, vacuumpacked and after $24 \mathrm{~h}$ were frozen $\left(-18{ }^{\circ} \mathrm{C}\right)$ for later chemical and sensory analysis.

\section{Chemical analysis}

Chops from the ninth and tenth ribs were taken from each animal and homogenized in a food processor (DPA2; Moulinex, Ecually, France). Moisture was analyzed using at oven at $85^{\circ} \mathrm{C}$ for $24 \mathrm{~h}$. Crude protein was analyzed using the Kjeldahl method, and IMF was extracted using a 2:1 chloroform-methanol mixture in a gravimetric system (Soxtec Foss Tecator 2055) adapting AOAC method $991^{(24,25)}$.

\section{Sensory evaluation}

Pork chop consumer acceptance was measured following the method of Pedrero(26). The panel consisted of 60 consumers (30 men, 30 women; age $=20$ to $45 \mathrm{yr}$ ) at the Faculty of Veterinary Medicine and Zootechny of the Autonomous University of the State of Mexico. Before the sensory trials, panelists were given precise instructions on how to qualify the pork and fill out the hedonic scale formats $(9=$ like very much; $5=$ neither like nor dislike; $1=$ dislike very much). Evaluated variables were juiciness, texture, flavor and aroma. After this orientation, the trial was held beginning at $1100 \mathrm{~h}^{(27)}$. Samples were prepared by defrosting the eleventh and twelfth chops from each animal for $20 \mathrm{~h}$ under refrigeration $\left(4{ }^{\circ} \mathrm{C}\right)$. They were then kept in darkness until reaching 16 ${ }^{\circ} \mathrm{C}$, the subcutaneous fat trimmed and chops cooked following AMSA techniques(28). After cooking, samples were kept in plastic bags at a constant temperature $\left(23^{\circ} \mathrm{C}\right)$ in a water bath. Samples were cut into cubes (approx. $1 \mathrm{~cm}^{3}$ ) and each panelist was offered two plates with two treatments per plate; each treatment was represented by two cubes. Assignment was done randomly and treatments 
alcanzaron $16{ }^{\circ} \mathrm{C}$; después de esto, la grasa periférica fue eliminada de las chuletas y se cocinaron según la metodología de $\operatorname{AMSA}^{(28)}$. Las muestras se mantuvieron a temperatura constante en bolsas de plástico, en baño María $\left(23{ }^{\circ} \mathrm{C}\right)$. A continuación, se cortaron en cubos de aproximadamente $1 \mathrm{~cm}^{3}$. A cada panelista se le ofrecieron dos platos con dos tratamientos cada uno (dos cubos de carne de cada tratamiento). Cada panelista recibió además agua, servilletas y galletas sin sal, y tuvo a su disposición un formato para rellenar en una escala hedónica de nueve puntos, donde $9=$ me gusta extremadamente mucho; $5=\mathrm{ni}$ me gusta ni me disgusta; y $1=$ me disgusta extremadamente(29). Las muestras fueron asignadas al azar a los panelistas y se identificaron con números aleatorios de tres dígitos. Después de evaluar las muestras de carne, cada panelista comió una galleta para eliminar los efectos de sabor, olor y aroma de cada muestra probada, y usó agua para enjuagarse la boca.

\section{Análisis estadístico}

Para la evaluación de la composición química de las chuletas, los datos se analizaron mediante un diseño experimental completamente al azar, con cuatro Tratamientos (dietas) y 12 repeticiones (unidades experimentales) por tratamiento, $(n=48)$. La información generada se analizó mediante los procedimientos GLM y Proc Mixed(30) del SAS. Los datos de la evaluación sensorial, se analizaron mediante una prueba de Kruskal-Wallis ${ }^{(31)}$ y para identified with three random numbers. Panelists also received water, salt-free crackers and a napkin. After evaluating a sample, the panelists ate a cracker to eliminate the flavor and aroma effects, and drank water to clean out their mouths.

\section{Statistical analysis}

Chemical composition was analyzed with a complete random experimental design consisting of four treatments (i.e. diets) and twelve replicates (i.e. experimental units) per treatment $(n=48)$. Data were analyzed with the GLM and Proc Mixed procedures of the SAS statistical package(30). Sensory evaluation data were analyzed with a Kruskal-Wallis test ${ }^{(31)}$ and general means generated to facilitate interpretation(30).

\section{RESULTS AND DISCUSSION}

Longissimus dorsi chemical composition (Table 3) was similar to previous reports $(17,32)$. Both moisture and protein content were independent $(P>0.3)$ of RAC use and dietary lysine level. In a study of pigs fed diets containing 12 to $20 \%$ protein, loin protein content varied up to nine percentile units $(33)$. These same diets produced increased pork juiciness and softness in the animals fed the low protein diet, perhaps due to a $60 \%$ increase in $\operatorname{IMF}^{(33)}$.

Chop IMF differed $(P<0.01)$ between treatments in response to lysine levels and RAC use. Pigs in the RLL treatment had $50 \%$ more IMF than those in the $\mathrm{C}$ treatment (Table 3), a difference equivalent

Cuadro 3. Composición química de la carne de cerdos alimentados con distintas concentraciones de lisina y ractopamina (\%)

Table 3. Chemical composition of pork from pigs fed experimental diets containing different lysine and ractopamine concentrations, and a control diet (\%)

\begin{tabular}{lcccccc}
\hline Variable & $\mathrm{C}$ & $\mathrm{RHL}$ & $\mathrm{RNL}$ & $\mathrm{RLL}$ & $\mathrm{SME}$ & $P \leq$ \\
\hline Moisture & 72.89 & 72.98 & 72.38 & 72.30 & 0.261 & 0.31 \\
Protein & 24.36 & 24.00 & 24.51 & 23.81 & 0.402 & 0.350 \\
Fat & $2.45 \mathrm{~b}$ & $2.65 \mathrm{~b}$ & $2.92^{\mathrm{b}}$ & $3.76 \mathrm{a}$ & 0.810 & 0.006 \\
\hline
\end{tabular}

$\mathrm{C}=$ Control, normal lysine level; $\mathrm{RHL}=$ ractopamine, high lysine level; $\mathrm{RNL}=$ ractopamine, normal lysine level; $\mathrm{RLL}=$ ractopamine, low lysine level; $\mathrm{SME}=$ standard mean error. 
facilitar su interpretación, se presentan las medias generales $(30)$.

\section{RESULTADOS Y DISCUSIÓN}

La composición química del músculo Longissimus dorsi (Cuadro 3), fue similar a lo reportado en estudios previos $(17,32)$. Tanto el contenido de humedad, como de proteína fueron independientes $(P>0.3)$ del uso de Ractopamina, o del nivel de lisina en la dieta. Estudios realizados en cerdos con dietas cuyo contenido de proteína varió de 12 a $20 \%$, muestran que el contenido de proteína en el lomo puede variar hasta en 9 unidades porcentuales $(33)$. Estos autores también indican un aumento en la jugosidad y la suavidad de la carne de los cerdos que consumieron la dieta baja en proteína, lo cual pudo ser debido a un aumento del $60 \%$ en la $\operatorname{GIM}^{(33)}$.

El contenido de GIM en las chuletas fue diferente $(P<0.01)$ entre tratamientos, por efecto del nivel de lisina y el uso de RAC. Las chuletas de los cerdos que consumieron la dieta RBL tuvieron $50.0 \%$ más GIM que los que recibieron la dieta $\mathrm{C}$ (Cuadro 3 ), lo que equivale a un incremento de 1.3 unidades porcentuales. Esto coincide con lo reportado por Cisneros(9) y por Witte(34), quienes lograron aumentar en 2 unidades porcentuales la GIM luego de alimentar a los cerdos, durante cinco semanas, con dietas equivalentes a las usadas en este experimento.

En general, el efecto de incluir la RAC en la dieta, independientemente del nivel de lisina, fue el de aumentar $26 \%$ la GIM sobre la dieta testigo $(P<0.01)$; lo que coincide con otros resultados $(16,35)$, donde se observó un mayor contenido de GIM en la carne de cerdos suplementados con RAC (10 ppm) en dietas con $16 \%$ de proteína cruda. Sin embargo, en otras investigaciones no se observaron efectos en la composición química de la carne de cerdos alimentados con dietas con más de $16 \%$ de PC y con niveles más elevados de RAC (dieta con 20 ppm RAC y $17 \%$ de PC) ${ }^{(36)}$; (dieta con 10 ppm to 1.3 percentile units. These results agree with previous reports from Cisneros ${ }^{(9)}$ and $\mathrm{Witte}^{(34)}$, who achieved a two percentile unit increase in IMF in pigs fed for 5 wk using equivalent diets to those presented in this study.

Inclusion of RAC in the diet, independent of lysine, had the overall effect of increasing IMF by $26 \%$ compared to that of the in the control diet $(P<0.01)$. This agrees with studies in which IMF increased in pigs fed RAC (10 ppm) and had CP (16\%) levels higher than those used here $(16,35)$. However, in other studies no changes in pork chemical composition were observed in pigs fed diets higher RAC and CP levels: 20 ppm RAC, $17 \% \mathrm{CP}^{(36)}$; 10 ppm RAC, $16 \% \mathrm{CP}^{(37)}$; 10 ppm RAC, $17 \%$ $\mathrm{CP}(38)$.

Crude protein levels used in the present study were lower than those recommended by the RAC manufacturer, mainly in an effort to maintain net diet energy contribution. Reducing dietary protein concentration also reduces the energy expenditure associated with nitrogen excretion, consequently raising net energy levels available for tissue growth, be it fat or muscle(39). The response to the lower dietary protein level used here may explain why no differences in IMF due to RAC levels were found in previous studies, including a meta-analysis of the RAC response ${ }^{(20)}$.

No differences $(P>0.17)$ between treatments were observed for meat aroma, flavor and texture (Table 4). This concurs with other studies in which no relationship could be found between IMF level and quality characteristics, using either instruments or trained panels $(40,41)$. However, it disagrees with studies in which differences in meat quality have been associated with higher $\operatorname{IMF}$ deposits $(1,2)$.

The RLL treatment produced the juiciest chops $(P<0.02)$, and tended to have the best flavor $(P<0.2)$. Based on previous observations, this could be associated with higher IMF levels $(1,2)$. A certain amount of IMF is considered essential for cooking and maintaining good meat quality(45). Fat 
Cuadro 4. Efecto de la concentración de lisina y de la adición de ractopamina en las características sensoriales de la carne de cerdo ${ }^{£}$

Table 4. Effect of lysine concentration and addition of ractopamine on pork chop sensory characteristics ${ }^{£}$

\begin{tabular}{lllllc}
\hline Variable & $\mathrm{C}$ & $\mathrm{RHL}$ & $\mathrm{RNL}$ & $\mathrm{RLL}$ & $P \leq$ \\
\hline Aroma & $5.60 \pm 1.2$ & $5.78 \pm 1.5$ & $5.67 \pm 1.5$ & $5.81 \pm 1.7$ & 0.562 \\
Juiciness & $6.08 \pm 1.4 \mathrm{~b}$ & $6.61 \pm 1.6 \mathrm{~b}$ & $6.37 \pm 1.5 \mathrm{~b}$ & $6.91 \pm 1.4 \mathrm{a}$ & 0.015 \\
Flavor & $6.06 \pm 1.4$ & $6.46 \pm 1.6$ & $6.26 \pm 1.6$ & $6.63 \pm 1.4$ & 0.170 \\
Texture & $6.38 \pm 1.5$ & $6.62 \pm 1.4$ & $6.41 \pm 1.6$ & $6.83 \pm 1.5$ & 0.428 \\
\hline
\end{tabular}

£Results of Kruskall-Wallis means comparison test for pork samples. Values are means \pm standard deviation. $\mathrm{C}=$ Control, normal lysine level; $\mathrm{RHL}=$ ractopamine, high lysine level; $\mathrm{RNL}=$ ractopamine, normal lysine level; $R L L=$ ractopamine, low lysine level.

abMeans with distint letters are different $(P<0.05)$.

RAC y $16 \%$ de PC) ${ }^{(37)}$; (dieta con 10 ppm RAC y $17 \%$ de PC) $(38)$.

En este experimento se formularon las dietas con niveles de proteína cruda inferiores a los recomendados por el fabricante de RAC, buscando proteger, principalmente, el aporte de energía neta de las dietas. Al reducir la concentración dietaria de proteína, se reduce el gasto energético asociado a la excreción de nitrógeno y por lo tanto, se incrementan los niveles de energía neta disponibles para el crecimiento de tejidos, ya sea en forma de grasa o de músculo(39). Por lo tanto, es factible que el nivel de proteína dietaria utilizado en este experimento, pueda explicar por qué en trabajos como el meta-análisis realizado por $\operatorname{Apple}^{(20)}$, no se encontraron diferencias en la GIM por efecto de RAC.

Con respecto a la evaluación sensorial de la carne, para las variables aroma, sabor y textura, el panel de consumidores no detectó diferencias $(P>0.17)$ entre las chuletas de los distintos tratamientos (Cuadro 4). Resultados similares han sido reportados por otros investigadores $(40,41)$, quienes no pudieron relacionar la cantidad de GIM con características de calidad, medidas instrumentalmente o con paneles entrenados. Sin embargo, otros estudios $(1,2)$ detectaron diferencias en la calidad de carne asociadas a un mayor depósito de GIM. stimulates saliva secretion in the mouth, meaning meat with higher IMF tends to be juicier(3). This suggests that the differences in juiciness observed in the present study may be attributed to higher IMF content.

Studies aimed at increasing meat production, usually evaluate the effect of high lysine and protein concentrations, which tend to increase feed efficiency. Unfortunately, high lysine and protein levels have an inverse effect on meat quality, producing a linear decrease in IMF and an increase in loin shear force $(17,42)$. For example, diets containing RAC and a high lysine concentration (2.4 or $3.1 \mathrm{~g}$ lysine/Mcal) reduced IMF and raised shear force(43). In another study(44), the use of diets containing $17.7 \%$ protein and $3 \mathrm{~g}$ lysine/ Mcal, showed that over time RAC resulted in a linear decline in marbling and the amount of fat chemically extracted from the loin. In contrast in this experiment, the $1.7 \mathrm{~g}$ lysine/Mcal included in the RLL treatment was associated with higher IMF deposition.

\section{CONCLUSIONS AND IMPLICATIONS}

Pork intramuscular fat content increased by $25 \%$ in response to addition of ractopamine (5 ppm) to diets containing $14.5 \%$ protein, which were fed to pigs for $28 \mathrm{~d}$ during the finishing period. An even 
Estudios enfocados a incrementar la productividad en función de la cantidad de carne producida, normalmente evalúan el efecto de concentraciones elevadas de proteína y lisina, ya que tienden a optimizar la eficiencia alimenticia. Desafortunadamente, los niveles elevados de lisina y proteína, tienen el efecto inverso en calidad de la carne, ya que tienden a reducir linealmente la GIM y a aumentar la fuerza de corte del lomo(17,42). Por ejemplo, Apple(43) encontró que en dietas con RAC y una elevada concentración de lisina (2.4 ó $3.1 \mathrm{~g}$ lisina/Mcal), se redujo la GIM y aumentó la fuerza de corte. Kutzler ${ }^{(44)}$ reportó que con el uso de dietas con niveles de $17.7 \%$ de proteína y $3 \mathrm{~g}$ de lisina/Mcal, el impacto del uso de RAC a lo largo del tiempo, se manifestó en una reducción lineal del marmoleo y de la cantidad de grasa extraída químicamente del lomo. En el presente estudio, la dieta RBL tuvo una concentración de $1.7 \mathrm{~g}$ lisina/ Mcal, lo que se asoció a una mayor infiltración de GIM.

La carne proveniente de animales consumiendo la dieta RBL, resultó más jugosa $(P<0.02)$, y mostró una tendencia a ser la de mejor sabor $(P<0.2)$, lo cual (basados en observaciones previas) pudiera estar asociado a la mayor cantidad de $\operatorname{GIM}^{(1,2)}$.

Cierta cantidad de GIM se considera esencial para cocinar y mantener una buena calidad de carne ${ }^{(45)}$. $\operatorname{Wood}^{(3)}$ indica que la grasa estimula los receptores de secreción de saliva en el consumidor, por lo tanto, la carne con mayor contenido de grasa intramuscular tiende a ser más jugosa. De acuerdo con esto, se puede mencionar que las diferencias observadas en la jugosidad, pueden ser atribuidas al mayor contenido de GIM observado en la carne.

\section{CONCLUSIONES E IMPLICACIONES}

Los resultados del estudio indican que la adición de RAC en dosis de 5 ppm, en dietas con un contenido de proteína de $14.5 \%$, ofrecidas durante los últimos 28 dias de la engorda, aumenta en $25 \%$ la grasa intramuscular de la carne de cerdo. Un incremento mayor (hasta el $50 \%$ ), se logra con higher intermuscular fat content (up to $50 \%$ ) was attained with a low lysine concentration (0.5\% standardized ileal digestible). Reductions in dietary protein and lysine contents in combination with the use of ractopamine increased pork juiciness as judged by a consumer panel.

\section{ACKNOWLEDGMENTS}

The authors thank PAIEPEME, A.C. and the CENID-Fisiología, INIFAP, Querétaro, México, for their collaboration. The research reported here was financed by the SAGARPA-COFUPROCONACYT Fund (\#109127).

End of english version

dietas con una baja concentración de lisina $(0.5 \%$ lisina digestible ileal estandarizada). Una reducción en la proteína y lisina dietarias, combinado con el uso de ractopamina aumenta la jugosidad de la carne de cerdo, de acuerdo con lo manifestado por el grupo de consumidores.

\section{AGRADECIMIENTOS}

Los autores agradecen la colaboración del PAIEPEME, A. C. Así como el apoyo del Fondo Sectorial SAGARPA-COFUPRO-CONACYT \#109127.

\section{LITERATURA CITADA}

1. Fernandez X, Monin G, Talmant A, Mourot J, Lebret B. Influence of intramuscular fat content on the quality of pig meat -2 . Consumer acceptability of $\mathrm{m}$. Longissimus lumborum. Meat Sci 1999;53(1):67-72.

2. Teye GA, Sheard PR, Whittington FM, Nute GR, Stewart A, Wood JD. Influence of dietary oils and protein level on pork quality. 1. Effects on muscle fatty acid composition, carcass, meat and eating quality. Meat Sci 2006;73(1):157-165. 
3. Wood JD, Nute GR, Richardson RI, Whittington FM, Southwood O, Plastow G, Mansbridge R, da Costa N, Chang KC. Effects of breed, diet and muscle on fat deposition and eating quality in pigs. Meat Sci 2004;(67):651-667.

4. Brewer S, Jensen J, Sosnicki A, Field B, Wilson E, McKeith F. The effect of pigs genetics on palatability, color and physical characteristics of fresh loin chops. Meat Sci 2002;(61):249256.

5. Verbeke W, Van Oeckel MJ, Warnants N, Viaene J, Boucque $\mathrm{ChV}$. Consumer perception, facts and possibilities to improve acceptability of health and sensory characteristics of pork. Meat Sci 1999;(53):77-99

6. Cameron ND, Enser MB. Fatty acid composition of lipid in longissimus dorsi muscle of Duroc and British Landrace pigs and its relationship with eating quality. Meat Sci 1991;(29):295307.

7. Kerr BJ, McKeith FK, Easter RA. Effect on performance and carcass characteristics of nursery to finisher pigs fed reduced crude protein, amino acid-supplemented diets. J Anim Sci 1995;(73):433-440.

8. Goerl KF, Eilert SJ, Mandigo RW, Chen HY, Miller PS. Pork characteristics as affected by two populations of swine and six crude protein levels. J Anim Sci 1995;(73):3621-3626.

9. Cisneros F, Ellis M, Baker DH, Easter RA, McKeith FK. The influence of short-term feeding of amino acid-deficient diets and high dietary leucine levels on the intramuscular fat content of pig muscle. J Anim Sci 1996;(63):517-522.

10. Witte DP, Ellis M, McKeith FK, Wilson ER. Effect of dietary lysine level and environmental temperature during the finishing phase on the intramuscular fat content of pork. J Anim Sci 2000;(78):1272-1276.

11. Castañeda-Silva EO, Cuarón IJA. Lysine to protein rations in growing-finishing pork [abstract]. J Anim Sci 2001;79(Suppl 1):321.

12. NRC. National Research Council. Metabolic Modifiers: Effects on the Nutrient Requirements of Food-Producing Animals. Washington, DC, USA: National Academy Press; 1994.

13. See MT, Armstrong TA, Matzat PD, Belstra BA, McKeith FK, Rincker PJ, Xi L, Odle J, et al. Effect of ractopamine feeding level on growth performance and carcass composition [abstract]. J Anim Sci 2005;83(Suppl 2):27.

14. Smith DJ. The pharmacokinetics, metabolism, and tissue residues of beta-adrenergic agonists in livestock. J Anim Sci 1998;76(1):173-194

15. Spurlock ME, Cusumano JC, Ji SQ, Anderson DB, Smith CK, Hancock DL, Mills SE. The effect of ractopamine on badrenoceptor density and affinity in porcine adipose and skeletal muscle tissue. J Anim Sci 1994;(72):75-80.

16. Watkins LE, Jones DJ, Mowrey DH, Anderson DB. The effect of various levels of ractopamine hydrochloride on the performance and carcass characteristics of finishing swine. $\mathrm{J}$ Anim Sci 1990;(68):3588-3595.

17. Webster MJ, Goodband RD, Tokach MD, Nelssen JL, Dritz SS, Unruh JA, Brown DA, et al. Interactive effects between ractopamine hydrochloride and dietary lysine on finishing pig growth performance, carcass characteristics, pork quality, and tissue accretion. Prof Anim Sci 2007;(23):597-611.
18. Mitchell AD, Solomon MB, Steele NC. Influence of level of dietary protein or energy on effects of ractopamine in finishing swine. J Anim Sci 1991;(69):4487-4495.

19. Williams NH, Cline TR, Schinckel AP, Jones DJ. The impact of ractopamine, energy intake, and dietary fat on finisher pork growth performance and carcass merit. J Anim Sci 1994;(72):3152-3162.

20. Apple JK, Rincker PJ, McKeith FK, Carr SN, Armstrong TA, PAS, Matzat PD. Meta-analysis of the ractopamine response in finishing swine. J Anim Sci 2007;(23):179-196.

21. NRC. National Research Council. Nutrient requirements of swine. $10^{\text {th }}$ ed. Washington, DC, USA; 1998.

22. Fernández DDM, Rosas VN, Pérez MVG, Cuarón IJA. Niveles de lisina digestible para cerdos finalizados con ractopamina [resumen]. XXXVII Congreso AMVEC. Puerto Vallarta, Jal. México. 2002:135.

23. Baker DH. Ideal amino acid profiles for swine and poultry and their applications in feed formulation. Biokyowa Tech Rev 9 Nutri-Quest Inc 1997;1-21.

24. AOAC. Official methods of analysis. $15^{\text {th }}$ ed. Arlington, VA, USA: Association of Official Analytical Chemists Inc. 1990.

25. Mariezcurrena MA, Braña D, Partida JA, Ramírez E, Domínguez IA. Estandarización de la metodología para la determinación de grasa en la carne de cerdo. Rev Mex Cienc Pecu 2010;1(3):269-275.

26. Pedrero FDL, Pangborn RM. Evaluación Sensorial de los Alimentos: Métodos Analíticos. México, DF: Alhambra Mexicana; 1989.

27. Anzaldua MA. La evaluación sensorial de los alimentos en la teoría y la práctica. Zaragoza, Esp: Acribia; 1994.

28. AMSA. American Meat Science Association. Research Guidelines for cookery and sensory evaluation and instrumental tenderness measurements of fresh meat. Chicago, Il, USA: Am Meat Sci Assoc. 2000.

29. Peryam DR, Pilgrim FH. Hedonic scale method for measuring food preferences. Food Tech 1957;11(9) 9-14.

30. SAS. SAS User's guide: Statistics (version 8 ed.) NC, USA: SAS Inst. Inc. 2006

31. Kruskal WH, Wallis WA. Use of ranks on one criterion variance analysis. J Am Stat Assoc 1952;(47):583-621.

32. Johnson LP, Miller MF, Haydon KD, Reagan OJ. The prediction of percentage of fat in pork carcasses. J Anim Sci 1990;(68):4185-4192.

33. Cromwell GL, Hays VW, Trujillo-Figueroa V, Kemp JD. Effects of dietary protein and energy levels for growing-finishing swine on performance, muscle composition and eating quality of pork. J Anim Sci 1978;(47):505-513.

34. Witte DP, Ellis M, McKeith FK, Wilson ER. Effect of dietary lysine level and environmental temperature during the finishing phase on the intramuscular fat content of pork. J Anim Sci 2000;(78):1272-1276.

35. Aalhus JL, Jones SDM, Schaefer AL, Tong AKW, Robertson WM, Merrill JK, Murray AC. The effect of ractopamine on performance, carcass composition and meat quality of finishing pork. Can J Anim Sci 1990;(70):943-952. 
36. Adeola O, Darko EA, He P, Young LG. Manipulation of porcine carcass composition by ractopamine. J Anim Sci 1990;(68):3633-3641.

37. Crome PK, McKeith FK, Carr TR, Jones DJ, Mowrey DH, Cannon JE. Effect of ractopamine on growth performance, carcass composition and cutting yields of pork slaughtered at 107 and 125 kilograms. J Anim Sci 1996;(74):709-716.

38. Stoller GM, Zerby HN, Moeller SJ, Baas TJ, Johnson C, Watkins LE. The effect of feeding ractopamine (Paylean) on muscle quality and sensory characteristics in three diverse genetic lines of swine. J Anim Sci 2003;(81):1508-1516.

39. van Milgen J, Noblet J. Partitioning of energy intake to heat, protein, and fat in growing pigs. J Anim Sci 2003;(81):E86E93.

40. Judge MD, Cahill VR, Kunkle LE, Deatherage FE. Pork quality II: physical, chemical and organoleptic relationships in fresh pork. J Anim Sci 1985;(36):145-149.
41. Rincker PJ, Killefer J, Ellis M, Brewer MS, McKeith FK. Intramuscular fat content has little influence on the eating quality of fresh pork loin chops. J Anim Sci 2008;(86):730-737.

42. Boler DD, Kutzler LW, Meeuwse DM, King VL, Campion DR, McKeith FK, Killefer J. Effects of increasing lysine levels on carcass composition and cutting yields of immunologically castrated male pigs. J Anim Sci 2011;89(7):2189-2199.

43. Apple JK, Maxwell CV, Brown DC. Effects of dietary lysine and energy density on performance and carcass characteristics of finishing pigs fed ractopamine. J Anim Sci 2004;(82):32773287.

44. Kutzler LW, Holmer SF, Boler DD, Carr SN, Ritter MJ, Parks $\mathrm{CW}$, McKeith FK, Killefer J. Comparison of varying doses and durations of ractopamine hydrochloride (Paylean $\left.{ }^{\circledR}\right)$ on late finishing pig carcass characteristics and meat quality $\mathrm{J}$ Anim Sci 2011;(89)7:2176-2188.

45. Dransfield E. The taste of fat. Meat Sci 2008;(80):37-42. 
